\title{
Avantages d'une configuration de machine à absorption opérant à trois niveaux de pression
}

\author{
Nahla Bouaziz ${ }^{a}$, Ridha Ben Iffa et Lakhdar Kairouani \\ Unité de Recherche Energétique \& Environnement, ENIT, Tunisia, École nationale d'Ingénieur de Tunis, \\ Université de Tunis El Manar, BP 37, 1002 le Belvédère, Tunisia
}

Reçu le 3 janvier 2011, accepté le 10 février 2011

\begin{abstract}
Résumé - Cette étude présente une machine frigorifique fonctionnant suivant un cycle à compressionabsorption. Le système proposé utilise le couple ammoniac-eau comme fluide frigorigène et opère à trois niveaux de pression. L'absorbeur fonctionne à une pression intermédiaire $P_{\text {int }}$, prise entre la pression de l'évaporateur (PEV) et celle du condenseur (PCD). Les principaux éléments du système proposé sont modélisés. Les résultats de la simulation numérique sont comparés à ceux relatifs au système conventionnel. L'étude comparative met en évidence les avantages du système objet de cette étude. Elle prouve le double avantage de la pression intermédiaire non seulement dans l'amélioration des performances de la machine à absorption mais aussi dans la réduction de la température du fonctionnement du générateur. Nous avons montré que pour des températures d'évaporation et de condensation prises respectivement à $-10{ }^{\circ} \mathrm{C}$ et $40{ }^{\circ} \mathrm{C}$, le cycle frigorifique fonctionne à une température du générateur TGE $=75{ }^{\circ} \mathrm{C}$ avec un COP de l'ordre de 0,56. La présente étude révèle que pour les mêmes conditions de fonctionnement, la machine frigorifique simple étage, présente un COP qui ne dépasse pas 0,51 avec une température du générateur de l'ordre de $135^{\circ} \mathrm{C}$. Le système hybride présente ainsi le privilège de fonctionner à basse température et de présenter des performances relativement améliorées.
\end{abstract}

Mots clés : Absorption / ammoniac / réfrigération / COP

\begin{abstract}
Advantages of a novel configuration of absorption cooling system operating at three pressure levels. The present study deals with a compression-absorption machine. The proposed hybrid system operates with water-ammonia and functions at three pressure levels. The absorber operates at an intermediate pressure $P_{\text {int }}$ taken between the evaporator pressure $(\mathrm{PEV})$ and the condenser pressure (PCD), unlike the one stage machine which functions at two level pressures. The system object of this work is examined and compared to the conventional cycle. The analyses of the numerical results highlight that the coefficient of performance of the novel proposed configuration is better than that relative to the simple effect absorption machine and reveals the great importance of the intermediate pressure not only in the performance improvement but also on reducing the generator temperature allowing the system to operate at low enthalpy sources. We showed for an evaporator temperature and a condenser temperature fixed respectively to $-10{ }^{\circ} \mathrm{C}$ and $40{ }^{\circ} \mathrm{C}$, the proposed hybrid refrigeration cycle, operates at a generator temperature $\mathrm{TGE}=75^{\circ} \mathrm{C}$ with an installation's $\mathrm{COP}$ about 0.56 .
\end{abstract}

Key words: Absorption / ammonia / refrigeration / COP

\section{Introduction}

L'industrie du froid a des répercussions importantes sur l'environnement $[1,2]$. Il importe que des mesures spécifiques à ce secteur soient prises pour maîtriser la consommation énergétique des installations frigorifiques de manière à limiter les rejets de gaz à effet de serre.

\footnotetext{
a Auteur pour correspondance :

nahlabouaziz@yahoo.fr
}

Les problèmes concernent principalement l'efficacité des installations et l'adaptation de ces systèmes aux nouvelles sources de chaleurs et à la recherche de nouveaux fluides frigorigène [3]. Les machines frigorifiques à absorption peuvent produire du froid en échangeant de la chaleur avec trois sources. Dans le présent travail, un intérêt particulier est accordé à l'optimisation des performances des systèmes à absorption et ce en cherchant une nouvelle configuration de cycle frigorifique permettant à la fois d'économiser l'énergie consommée et d'assurer un 


\section{Nomenclature}

\begin{tabular}{|lll|}
\hline Symbole & Quantité & SI Unit \\
\hline COP & Coefficient de performance & \\
$f$ & Facteur de circulation & \\
$h$ & Enthalpie specifique & $\left({\left.\mathrm{J} . \mathrm{kg}^{-1}\right)}^{-1}\right.$ \\
$\dot{m}$ & Débit masse & $\left(\mathrm{kg} \cdot \mathrm{s}^{-1}\right)$ \\
$P$ & Pression & $(\mathrm{bar}, \mathrm{Pa})$ \\
$\dot{Q}$ & Puissance & $(\mathrm{W})$ \\
$T$ & Température & $\left(\mathrm{K},{ }^{\circ} \mathrm{C}\right)$ \\
$x$ & Fraction de masse & \\
$\mathrm{Subscripts}$ & & \\
$\mathrm{AB}$ & Absorbeur & \\
$\mathrm{CD}$ & Condenseur & \\
$\mathrm{COM}$ & Compresseur & \\
$\mathrm{EV}$ & Évaporateur & \\
$\mathrm{GE}$ & Generateur & \\
$\eta$ & Efficacité $\%$ & \\
\hline
\end{tabular}

coefficient de performance amélioré $[4,5]$. Nous proposons dans cette étude une nouvelle machine frigorifique fonctionnant suivant un cycle à compression-absorption et opérant à trois niveaux de pression.

\section{Modélisation du système}

Plusieurs auteurs ont étudié des modèles de machines à absorption utilisant les couples $\mathrm{H}_{2} \mathrm{O} / \mathrm{NH}_{3}$ et $\mathrm{LiBr} / \mathrm{H}_{2} \mathrm{O}$ comme fluides frigorigène et l'énergie géothermale et solaire comme source d'énergie [6-10]. La machine à absorption est composée par six éléments principaux évaporateur, absorbeur(s), pompe(s), générateur(s), condenseur et détendeur(s). Pour modéliser un cycle frigorifique à absorption, il suffit de modéliser le fonctionnement de chacun de ces composants.

\subsection{Modèle conventionnel simple étage}

Ce modèle est composé par un évaporateur, un absorbeur, une pompe, un générateur, un condenseur et deux détendeurs : un pour le retour de la solution pauvre à l'absorbeur et l'autre pour la détente du réfrigérant dans l'évaporateur et un échangeur inter-solution. On désigne par un modèle simple étage toute installation travaillant entre deux niveaux de pression : la pression de l'évaporateur (PEV) et la pression du condenseur (PCD) figure 1. Le COP théorique d'un tel système est calculé en considérant les hypothèses suivantes :

- la température au niveau de chaque composant principal est uniforme;

- la vapeur réfrigérante est en équilibre thermodynamique avec la solution riche entrant de l'absorbeur et la solution pauvre sortant du générateur;

- la solution pauvre quitte à la température du générateur et à l'équilibre thermodynamique ;

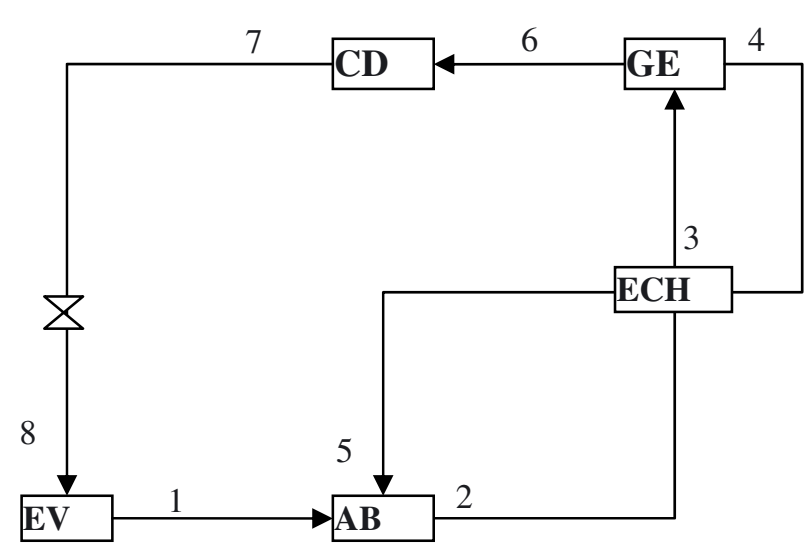

Fig. 1. Cycle conventionnel.

- le titre de vapeur quittant le générateur peut être considéré comme vapeur dont la concentration est inférieure à l'unité [11].

Dans le but de réduire la température de fonctionnement des systèmes frigorifiques à absorption $\left(\mathrm{NH}_{3}-\mathrm{H}_{2} \mathrm{O}\right)$, nous avons étudié plusieurs configurations afin de sélectionner celle qui peut opérer à des températures de générateur relativement basse. L'objet du présent travail, est une machine hybride. On se propose d'étudier ses performances et de les comparer au système conventionnel. La solution proposée permet de contourner les limites de fonctionnement du cycle classique tout en assurant un coefficient de performance supérieur à celui observé dans le cadre des machines à absorption simple effet.

Nous avons montré dans une étude ultérieure que le système à absorption classique utilisant le couple $\mathrm{NH}_{3}-$ $\mathrm{H}_{2} \mathrm{O}$ ne peut pas fonctionner avec une température inférieure à $100{ }^{\circ} \mathrm{C}$ au niveau du générateur, pour une température de l'évaporateur de $-10^{\circ} \mathrm{C}$. De plus la valeur maximale du coefficient de performance est de l'ordre de $0,51[12]$.

\subsection{Modèle évapo-compression}

La figure 2 présente la nouvelle configuration de machine à absorption proposée dans le cadre de ce travail. Cette machine est composée d'un évaporateur, un absorbeur, un générateur, un condenseur et un compresseur inséré entre l'évaporateur et l'absorbeur qui a pour rôle d'amener la vapeur à la sortie de l'évaporateur vers une pression intermédiaire (PAB).

\section{Générateur GE}

La solution riche en frigorigène (4) reçoit la quantité de chaleur QGE à une température TGE ce qui provoque la vaporisation d'une partie du frigorigène dissous dans la solution. On parle alors de désorption. À la sortie du générateur, on obtient donc de la vapeur du frigorigène (6) et une solution appauvrie en frigorigène (5). 


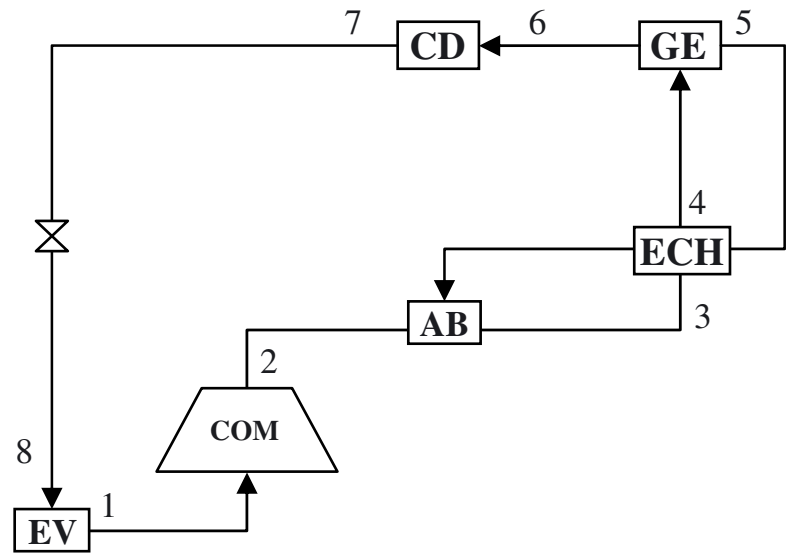

Fig. 2. Cycle évapo-compression.

\section{Condenseur $C D$}

Il s'agit de composant analogue à celui des machines à compression de vapeur. C'est la température TCD du fluide caloporteur alimentant le condenseur qui fixe la température de condensation et donc la pression dans l'ensemble désorbeur/condenseur. La condensation du frigorigène nécessite le rejet de la chaleur de condensation QCD à la température TCD.

\section{Évaporateur EV}

À la sortie du condenseur (7), le liquide subit un laminage à travers le détendeur (9), puis s'évapore en prenant la chaleur QEV du fluide ou à l'enceinte à refroidir. La température d'évaporation, et par suite la pression dans l'ensemble évaporateur/absorbeur est fixée par la température de la source froide TEV.

\section{Absorbeur $A B$}

La vapeur issue du compresseur (2) enrichit la solution dans l'absorbeur. On parle alors d'absorption. La chaleur QAB dégagée par cette transformation exothermique est évacuée par un fluide caloporteur à la température TAB. À la sortie de l'absorbeur (3), on obtient une solution enrichie en frigorigène.

\section{Compresseur COM}

À la sortie de l'évaporateur (1) la vapeur comprimée par un compresseur vers l'absorbeur. On soulève trois niveaux de pression : une basse pression $(\mathrm{BP}=\mathrm{PEV})$, une pression moyenne $(\mathrm{PM}=\mathrm{PAB})$ et une haute pression $(\mathrm{HP}=\mathrm{PCD}=\mathrm{PGE})$.

\subsection{Bilan d'énergie et de masse}

Le facteur d'entraînement représentant le débit de la solution riche permet de dégager un $\mathrm{kg}$ de $\mathrm{NH}_{3}$ du générateur :

$$
f=\frac{1-x_{5}}{x_{4}-x_{5}}
$$

En négligeant le rectifieur, les bilans d'énergie au niveau de chaque composant de l'installation, échangeant de la chaleur ou du travail avec le milieu extérieur sont représentés ci-après :

\section{Condenseur}

$$
\begin{aligned}
\dot{m}_{6} & =\dot{m}_{7}=\dot{m}_{\mathrm{NH}_{3}} \\
T_{7} & =T_{\mathrm{CD}} \\
h_{7} & =h\left(T_{\mathrm{CD}}\right) \\
P_{7} & =P\left(T_{\mathrm{CD}}\right)=P_{\mathrm{CD}} \\
\dot{Q}_{\mathrm{CD}} & =\dot{m}_{\mathrm{NH}_{3}}\left(h_{6}-h_{7}\right)
\end{aligned}
$$

\section{Évaporateur}

$$
\begin{aligned}
\dot{m}_{8} & =\dot{m}_{7}=\dot{m}_{\mathrm{NH}_{3}} \\
T_{1} & =T_{\mathrm{EV}} \\
h_{8} & =h_{7}, \quad h_{1}=h\left(T_{\mathrm{EV}}\right) \\
P_{8} & =P_{1}=P\left(T_{\mathrm{EV}}\right)=P_{\mathrm{EV}} \\
\dot{Q}_{\mathrm{EV}} & =\dot{m}_{\mathrm{NH}_{3}}\left(h_{1}-h_{8}\right)
\end{aligned}
$$

\section{Générateur}

$$
\begin{aligned}
\dot{m}_{4} & =\dot{m}_{6}+\dot{m}_{5} \\
P_{6} & =P_{5}=P_{4}=P_{\mathrm{CD}} \\
T_{6} & =T\left(x_{4}, P_{\mathrm{CD}}\right), \quad T_{5}=T_{\mathrm{GE}} \\
x_{5} & =x\left(T_{5}, P_{\mathrm{CD}}\right), \quad x_{4}=x_{5}+\Delta x \\
h_{6} & =h\left(x_{4}, P_{\mathrm{CD}}\right), \quad h_{5}=h\left(x_{5}, P_{\mathrm{CD}}\right) \\
\dot{Q}_{\mathrm{GE}} & =\dot{m}_{\mathrm{NH}_{3}}\left(h_{6}+(f-1) h_{5}-f h_{4}\right)
\end{aligned}
$$

\section{Compresseur}

À la sortie de l'évaporateur, la vapeur d'ammoniac est assimilée à un gaz parfait, par conséquent, la puissance est définie par :

$$
T_{\text {ent_comp }} \times P_{\text {ent_comp }}^{(k-1) / k}=T_{\text {sor_comp }} \times P_{\text {sor_comp }}^{(k-1) / k}
$$

Dans le cas idéal de processus adiabatique, supposé sans frottement, le système consomme une puissance :

$$
\dot{Q}_{\text {is }}=c p_{\mathrm{NH}_{3}} \times\left(T_{\text {sor_comp }}-T_{\text {ent_comp }}\right)
$$

En tenant compte du rendement isentropique $\eta_{\text {is }}$ la puissance réelle s'écrit :

$$
\dot{Q}_{\text {réel }}=\frac{\dot{Q}_{\text {is }}}{\eta_{\text {is }}}
$$

Avec :

$$
\dot{Q}_{\text {réel }}=h_{\text {sor_comp }}-h_{\text {ent_comp }}
$$

Les équations (20) et (21) permettent de déduire l'enthalpie vapeur à la sortie du compresseur :

$$
h_{\text {sor_comp }}=h_{\text {ent_comp }}+\frac{\dot{Q}_{\text {is }}}{\eta_{\text {is }}}
$$


L'expression du rendement est définie par :

$$
\begin{gathered}
\eta_{\text {is }}=0,874-0,0135 \tau \\
\text { et } \quad \tau=\frac{P_{\text {sortie }}}{P_{\text {entrée }}}
\end{gathered}
$$

Absorbeur

$$
\begin{gathered}
\dot{m}_{2}=\dot{m}_{3} \\
x_{3}=x_{2} \\
T_{3}=T_{\mathrm{CD}} \\
\dot{Q}_{\mathrm{AB}}=\dot{m}_{\mathrm{NH}_{3}}\left(h_{2}-h_{3}\right)
\end{gathered}
$$

Les équations (11) et (17) permettent de déduire le coefficient de performance :

$$
\mathrm{COP}=\frac{\dot{Q}_{\mathrm{EV}}}{\dot{Q}_{\mathrm{GE}}}=\frac{h_{1}-h_{2}}{h_{7}+(f-1) h_{5}-f h_{4}}
$$

\section{Résultats et discussion}

Nous avons développé un programme numérique. Les procédures de calcul des propriétés thermodynamiques des fluides et du coefficient de performance ont été établies en utilisant le logiciel MAPLE comme outils [12]. L'analyse des résultats de la simulation montre que pour une machine simple étage la variation du COP dépend de trois paramètres à savoir la température d'évaporation, température nécessaire pour la production du froid désiré, la température de condensation qui est fonction de la température du fluide disponible pour le refroidissement de l'absorbeur et du condenseur et enfin la température du générateur, source de chauffage disponible. Pour la nouvelle installation, un nouveau paramètre se révèle intéressant pour l'étude des performances du cycle frigorifique proposé dans cette étude, c'est la pression d'absorption intermédiaire $P_{\text {int }}$.

La figure 3 montre l'évolution du COP en fonction de la température du générateur en fixant respectivement la température de l'évaporateur à $-10^{\circ} \mathrm{C}$ et celle du condenseur à $40^{\circ} \mathrm{C}$, pour la machine à absorption classique et la nouvelle configuration du cycle à absorption, la pression d'absorption étant fixée à $650 \mathrm{kPa}$, pour la nouvelle installation.

L'analyse de la figure 3 montre que pour la nouvelle configuration de la machine à absorption, le COP est plus élevé, de l'ordre de 0,58, par contre le coefficient de performance du cycle conventionnel ne dépasse pas 0,51. D'autre part, la température de fonctionnement est relativement faible, autour de $70{ }^{\circ} \mathrm{C}$ par rapport à celle du cycle classique qui est de l'ordre de $105{ }^{\circ} \mathrm{C}$. Un gain d'énergie relativement important est ainsi mis en évidence même dans las conditions de fonctionnement les plus défavorables. En plus, même pour des températures du générateur élevées $\left(\mathrm{TGE}>105^{\circ} \mathrm{C}\right.$ ) le $\mathrm{COP}$ continue à être supérieur à celui du cycle conventionnel.

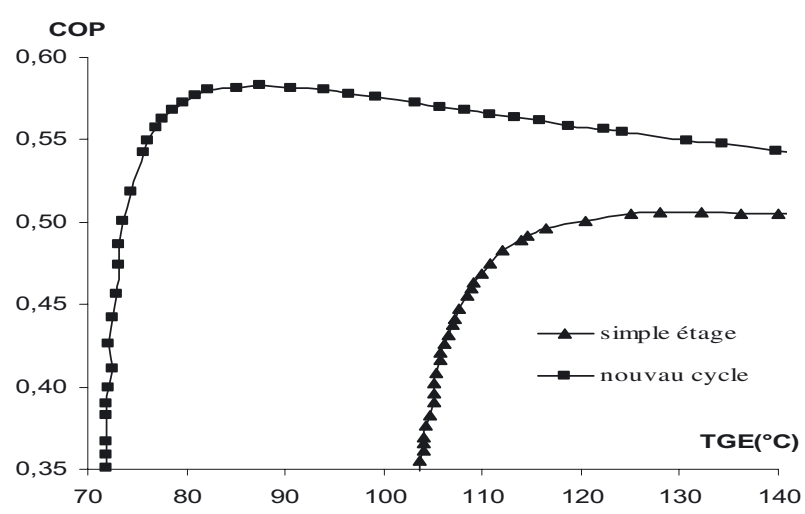

Fig. 3. $\mathrm{COP}=f(\mathrm{TGE})$ pour le système conventionnel et la configuration proposée.

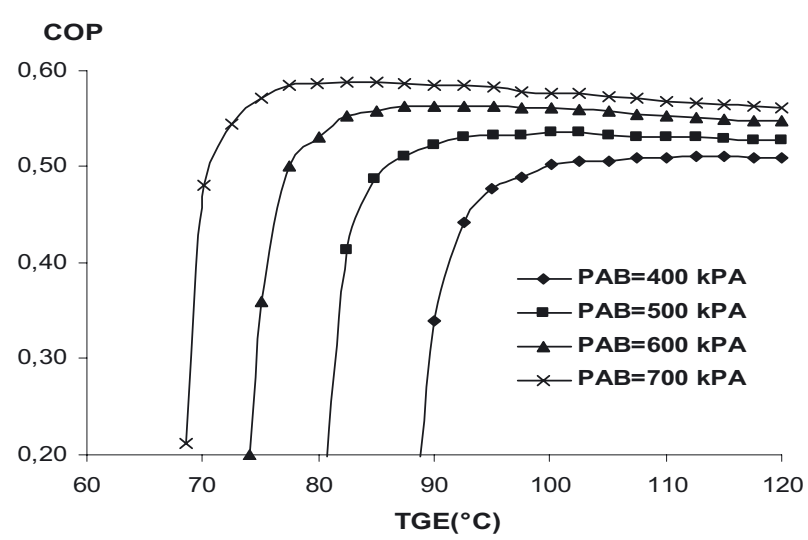

Fig. 4. $\mathrm{COP}=f(\mathrm{TGE})$ avec $\mathrm{TEV}=-10^{\circ} \mathrm{C}$ et $\mathrm{TCD}=40{ }^{\circ} \mathrm{C}$.

\subsection{Variation du COP en fonction de TGE}

Pour une température de condensation de $40^{\circ} \mathrm{C}$ et une température d'évaporation de $-10^{\circ} \mathrm{C}$, la figure 4 , présente une famille de courbes montrant la variation du coefficient de performance en fonction de la température du générateur allant de $68{ }^{\circ} \mathrm{C}$ jusqu'à $120^{\circ} \mathrm{C}$. On remarque que la nouvelle machine fonctionne sur tout le domaine de température et que son coefficient de performance croît si la pression intermédiaire augmente. En plus la température de fonctionnement (TGE) diminue si la pression de l'absorbeur augmente. L'analyse de ces courbes montre également que si l'absorbeur fonctionne à une pression supérieure à celle de l'évaporateur, le système frigorifique pourra fonctionner à une température plus basse au niveau du générateur permettant ainsi le fonctionnement du système à basse enthalpie.

\subsection{Variation du COP en fonction de TCD}

On fixe les températures du générateur et de l'évaporateur respectivement à $100^{\circ} \mathrm{C}$ et $5^{\circ} \mathrm{C}$, et on fait varier la température de condensation pour chacun des deux cycles. La figure 5 montre que le COP de la nouvelle installation, proposée dans le cadre de cette étude augmente avec la température de condensation. Par contre le 


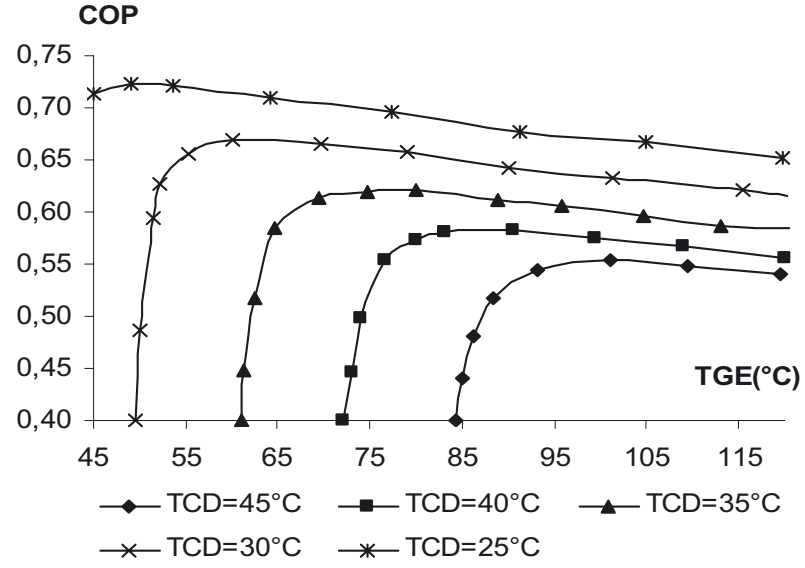

Fig. 5. $\mathrm{COP}=f(\mathrm{TGE})$ avec $\mathrm{TEV}=-10{ }^{\circ} \mathrm{C}$ et $\mathrm{PAB}=$ $650 \mathrm{kPa}$.

COP de la machine à absorption conventionnelle diminue si la température de condensation croît.

\section{Conclusion}

Dans le présent travail, nous avons analysé le fonctionnement d'une nouvelle configuration d'une machine frigorifique à absorption. Le système proposé fonctionne à trois niveaux de pression et opère au couple ammoniaceau. Afin d'analyser les performances du cycle considéré, nous avons modélisé chacun de ses composants. Les résultats de la simulation numérique obtenus sont comparés à ceux relatifs au système conventionnel. Les quelques résultats présentés font preuve des avantages de la configuration objet de cette étude. La machine fonctionnant suivant un cycle hybride à compressionabsorption présente le privilège de fonctionner à faible valeur d'enthalpie et de présenter des performances relativement meilleurs que ceux de la machine frigorifique simple étage opérant entre deux niveaux de pression. En effet, l'introduction de la pression intermédiaire présente le double avantage de baisser la température de fonctionnement du générateur et d'améliorer le COP du système. Pour des températures d'évaporation et de condensation prises respectivement égales à $-10{ }^{\circ} \mathrm{C}$ et $40{ }^{\circ} \mathrm{C}$, nous avons montré que la machine fonctionne à une température du générateur TGE $=75{ }^{\circ} \mathrm{C}$ avec un COP de l'installation de l'ordre de 0,56. Pour les mêmes conditions, le cycle classique présente un COP qui ne dépasse pas 0,51 avec une température du générateur relativement élevée, de l'ordre de $135^{\circ} \mathrm{C}$. Les conséquences de cette investigation révèlent ainsi que l'introduction d'une pression intermédiaire est d'une importance capitale dans l'adaptation des cycles frigorifiques à absorption aux sources fonctionnant à basse température et par conséquent dans l'économie de l'énergie consommée dans le domaine de production de froid. Pour valider ces résultats, il suffira d'associer le système considéré à une source solaire et en particulier d'utiliser des capteurs solaires plans ordinaires peu coûteux et disponibles sur le marché.

\section{Références}

[1] A. Keçeciler, H.I. Acar, A. Dogan, Thermodynamic analysis of the absorption refrigeration system with geothermal energy: an experimental study, Energy Convers. Manag. 41 (2000) 37-48

[2] M. Thioye, Amélioration de la performance des machines frigorifiques à absorption par l'utilisation de cycles à absorption et désorption étagés, Int J. Refrig. 20 (1997) 136145

[3] Fathi, C. Guemimi, S. Ouaskit, An irreversible thermodynamic model for solar absorption refrigerator, Renewable Energy 29 (2004) 1349-1365

[4] A.T. Bulgan, Use of low temperature energy sources in aqua-ammonia absorption refrigeration systems, Energy Convers. Management 38 (1997) 1431-1438

[5] A. Laouir, P. legoff, J.M. Hornt, Cycle de frigopompes à absorption en cascades matérielles-détermination du nombre d'étages optimal pour le mélange ammoniac-eau, Int J. Refrig. 25 (2002) 136-148

[6] D. Boer, M. Valles, A. Coronas, Performance of double effect absorption compression cycles for air-conditioning using methanol-TEGDME and TFE-TEGDME systems as working pairs, Int J. Refrig. 21 (1998) 542-555

[7] M. Fukuta, T. Yanagisawaa, H. Iwatab, K. Tadab, Performance of compression/absorption hybrid refrigeration cycle with propane/mineral oil combination, Int J. Refrig. 25 (2002) 907-915

[8] Y.T. Kang, Y. Kunugi, T. Kashiwagi, Review of advanced absorption cycles : performance improvement and temperature lift enhancement, Int J. Refrig. 23 (2000) 388-401

[9] S. Göktun, Performance analysis of a heat engine driven combined vapor compression-absorption-ejector refrigerator, Energy Convers. Mgmt 41 (2000) 1885-1895

[10] S.C. Kaushik, K.T. Lam, S. Chandra, C.S. Tomar, Mass and energy storage analysis of an absorption heat pump with simulated time dependent generator heat input, Energy Convers. Mgmt 22 (1982) 183-196

[11] R.J. Romero, Luis Guillen, I. Pilatowsky, Monomethylamine-water vapour absorption refrigeration system, Appl. Th. Eng. 25 (2005) 867-876

[12] L. Kairouani, E. Nehdi, R. Ben Iffa, Thermodynamic investigation of two-stage absorption refrigeration system connected by a compressor, Am. J. Appl. Sci 2 (2005) 1036-1041 